\title{
Richard Edlin, Christopher McCabe, Claire Hulme, Peter Hall, Judy Wright: Cost Effectiveness Modelling for Health Technology Assessment: A Practical Course
}

\author{
2015 | Adis International Springer Cham Heidelberg New York, Dordrecht, London | \\ pp 208 | ISBN 978-3-319-15743-6
}

Doug Coyle ${ }^{1}$

Published online: 28 January 2016

(c) Springer International Publishing Switzerland 2016

Cost Effectiveness Modelling for Health Technology Assessment: A Practical Course by Edlin, McCabe Hulme, Hall and Wright is a new entry into the increasing field of textbooks relating to economic evaluation in health care. In the preface, the authors highlight that their intention was to provide a book that is accessible to most readers whilst providing an up-to-date reflection of the current best practices in cost effectiveness modelling.

Chapter 1 provides an overview of the rationale and analytical frameworks for cost effectiveness analysis in health care. The sections on dominance and extended dominance and the net benefit framework are of particular clarity and provide an exemplar explanation of the concepts.

Chapter 2 approaches the topic of finding evidence for cost effectiveness modelling. Focus is on systematic approaches to sourcing not just clinical evidence but underlying disease epidemiology, costs and utilities. This is an incredibly welcome addition often neglected in previous texts. Given the focus of the text as a practical course, this chapter may have been improved by a thorough description of a practical example of data elicitation for a particular study.

Chapter 3 introduces the focus of the text as a practical course in modelling. The concept of modelling is introduced, and an appropriate example of analysis using a decision tree is used as an illustration. Readers are taken through the construction and population of the decision tree

Doug Coyle

dcoyle@uottawa.ca

1 Doug Coyle School of Epidemiology, Public Health and Preventive Medicine, University of Ottawa, Ottawa, ON, Canada both "on paper" and through the use of an Excel workbook. One caution I have is that the level of the text and the exercise assumes a reasonable degree of experience working with Excel, which I have realized in the past is not always an appropriate assumption for all graduate students.

Chapter 4 is a well written summary of the methods of addressing uncertainty within cost effectiveness modelling. The discussion on sources of uncertainty is clear and valuable, whilst the review of deterministic methods is both clear and appropriately cautionary. Probabilistic analysis is introduced here but discussed in much more detail in Chapters 6-8 and 10-11.

Chapter 5 provides an introduction to Markov models. The chapter outlines the rationale for the adoption of such models and deftly introduces key concepts such as transition probabilities and Markov trace. Chapter 9 takes the concepts introduced in Chapter 5 and allows readers to follow the stepby-step construction of a Markov model in Excel.

It is in the chapters relating to probabilistic analysis that the value of the text comes to the fore. Chapters 6 and 7 provide an excellent discussion on choosing and constructing probability distributions for input parameters. Chapter 8's focus on incorporation of correlation between input parameters is a welcome addition which frequently has been given short shrift. Chapter 10 allows the reader to follow how the deterministic model from Chapter 9 can be made stochastic, with Chapter 11 providing the reader the technical tools to appropriately analyse and present the results of such analyses.

Chapters 12 and 13 provide well written summaries of the state of the art in terms of the application of modelling to decision making, with focus on the value of further information. These are accessible to most readers but could be avoided by those less technically minded readers. 
The text is designed to be a practical guide to the conduct of cost effectiveness modelling. Hence, throughout the text there are references to practical exercises relating to the methods discussed. The companion online workbook and practical exercises are well designed-although there are some initial disconnects between the text, workbook and exercises that I am sure will be addressed.

Given the focus on practice, the one possible weakness of the book is in many ways an intention of the authors. The text does not provide detailed theoretical and conceptual discussions about the methods adopted and the assumptions undertaken. The authors intended to introduce these issues in "bite-sized pieces" which allow certain readers to solely learn the methods employed, whilst others should be encouraged to review further materials for greater theoretical insights and discussions. Many methodological issues are not solved within this textbook (e.g. how to assume correlation between input parameters from disparate sources), but that may be too much to ask within this setting.

Cost Effectiveness Modelling for Health Technology Assessment: A Practical Course is an excellent addition to the increasing textbook options in the field of economic evaluation in health care. It fills an important gap in terms of a more practical focus on the application of the concepts and theoretical principles underlying the discipline. The text is well written, and the practical exercises provide insight into the conduct of modelling. It will be best used as either a stand-alone text for a short course for health care decision makers to understand the application of the discipline or as a companion text for introductory graduate courses in economic evaluation.

\section{Compliance with Ethical Standards}

Conflicts of interest D. Coyle has no conflicts of interest with respect to this review. 